\title{
Revisión del género Philonerax Bromley, 1932 (Diptera: Asilidae: Asilinae) y dos nuevas especies
}

\author{
Revision of the genus Philonerax Bromley, 1932 (Diptera: Asilidae: Asilinae) and \\ two new species
}

\author{
MARTHA B. HENGST ${ }^{1} \&$ JORGE N. ARTIGAS ${ }^{2}$
}

\begin{abstract}
${ }^{1}$ Centro de Biotecnología y Biología Molecular, Facultad de Recursos del Mar, Universidad de Antofagasta, Casilla 170, Antofagasta, Chile; e-mail: mbhengst@puc.cl

${ }^{2}$ Departamento de Zoología, Facultad de Ciencias Naturales y Oceanográficas, Universidad de Concepción, Casilla 160C, Concepción, Chile; e-mail: jartigas@udec.cl
\end{abstract}

\begin{abstract}
RESUMEN
El género Philonerax descrito por Bromley en 1932, fue sinonimizado a Lochmorhynchus Engel, 1930 por Artigas \& Papavero (1995). En este trabajo, Philonerax recupera su estatus de género; la especie Philonerax cribatus Hull es sinonimizada a Ph. mucidus (Walker) y se crean dos nueva especies: Ph. amblayoensis sp. nov. de Argentina y Ph. cienagaensis sp. nov. de Argentina y Bolivia; principalmente en base a caracteres de la genitalia de machos y hembras.
\end{abstract}

Palabras clave: Asilidae, Philonerax, Argentina, Bolivia, Chile.

\begin{abstract}
The genus Philonerax described by Bromley in 1932, was sinonimized to Lochmorhynchus Engel, 1930 by Artigas \& Papavero (1995). In this paper Philonerax recovers its generic status; the species Philonerax cribatus Hull was sinonimized to Ph. mucidus (Walker) and two new species were described: Ph. amblayoensis sp. nov. from Argentina and $P h$. cienagaensis from Argentina and Bolivia, based mainly on male and female genital characters.
\end{abstract}

Key words: Asilidae, Philonerax, Argentina, Bolivia, Chile.

\section{INTRODUCCIÓN}

El género Philonerax (Asilidae: Asilinae), fue creado por Bromley (1932), para la única especie Asilus mucidus Walker, 1837, de Argentina, Port Santa Elena $\left(37^{\circ} 21^{\prime} \mathrm{S}, 60^{\circ} 37^{\prime} \mathrm{O}\right)$. Estudió material de Argentina (cinco machos y dos hembras), Nahuelhuapi, Bariloche, Patagonia y Chubut.

Hull (1962) hace una extensa descripción del género y menciona la única especie mucidus con distribución Neotropical. Incluye figuras: 212 antena, 689 ala hembra; 705 posiblemente hembra; 750 ala macho; 1439 sp. indeterminada, cabeza vista lateral: $1448 \mathrm{sp}$. indeterminada, cabeza vista frontal; 2225 mucidus genitalia macho vista lateral. Crea en la misma publicación el género Lonchodogonus con una nueva especie, cribatus (pp. 496-498) sobre un holotipo y alotipo de procedencia desconocida: "Distribución: país desconocido, nueva especie. No hay etiqueta de localidad en estos ejemplares. El género más próximo relacionado es de Chile"..."se cree que son de la región de la cordillera del sur de Sud
América, de donde son todos los emparentados de estas moscas. En Zoologische Staatssammlung, Munich"...(traducido del inglés).

Artigas (1970) crea la especie chilechicoensis que diferencia de mucidus en una clave e incluye figuras: Ph. mucidus: 363 genitalia macho lateral; funda pene lateral; 365 tibia frontal. $\mathrm{Ph}$. chilechicoensis: 366 cabeza lateral, 367 genitalia macho lateral; 368 funda del pene lateral; 369 ovipositor dorsal; 370 ala macho. Además, sugiere que el género Lonchodogonus Hull, 1962, podría ser sinónimo de Philonerax. Artigas \& Papavero (1995) ubican a Philonerax Bromley y Lonchodogonus Hull como sinónimo de Lochmorhynchus Engel. En su trabajo para los géneros de asílidos argentinos, Artigas \& Hengst (1998), incluyen a Philonerax como sinónimo de Lochmorhynchus, manteniendo la propuesta de Artigas \& Papavero (1995). Hengst (2000) restablece la validez de Philonerax a la conocida especie mucidus Walker y le agrega dos nuevas especies: tibialis (amblayoensis en este trabajo) y sagitata (cienagaensis en este trabajo). En el 
mismo trabajo, determina que la especie Philonerax chilechicoensis Artigas, 1970 es sinónimo de mucidus.

Philonerax mucidus (Walker) es reconocible solo por la descripción original y las figuras disponibles. El carácter más destacado es el extremo del esternito 8, en el cual las proyecciones terminales se abren hacia los costados y se expanden en el ápice, donde llevan un tupido mechón de cerdas largas que se dirigen hacia el centro, donde se cruzan, cerrando la abertura posterior (Artigas \& Papavero 1995).

La especie Philonerax chilechicoensis Artigas 1970, es descrita con respecto al esternito 8 como: "pelos largos en las proyecciones laterales del esternito 8..." sin embargo en la figura 367 (genitalia vista lateral), no se observa que los brazos apicales del esternito 8 se extiendan lateralmente, asemejándose esta figura más a la nueva especie, Ph. amblayoensis propuesta en este trabajo. El holotipo macho de chilechicoensis guardado en el MZUC es claramente mucidus (esternito 8), por lo cual chilechicoensis es sinónimo de mucidus.

La especie Lonchodogonus cribatus Hull, 1962 "posiblemente del sur de Sudamérica" (Zool. Staatssamlung, Munich, no visto), según las figuras de Hull, 1962: 2256, 2281, 2373 y 2382, es claramente Ph. mucidus. Las figuras 209, 751, 1435 y 1444, son generales para el género Philonerax. Por ello cribatus Hull es sinónimo de mucidus Walker. Se concluye que Philonerax es un género válido con tres especies: mucidus Walker y dos especies nuevas para la ciencia, cienagaensis (sagitata en tesis de Hengst y amblayoensis (tibialis en tesis de Hengst), que se proponen en el presente trabajo.

\section{MATERIALES Y MÉTODOS}

Se revisaron 52 ejemplares depositados en las siguientes colecciones: Instituto Miguel Lillo, Tucumán, Argentina (IML); Museo Nacional de Historia Natural, Chile (MNHN): Smithsonian Institution, National Museum of Natural History, New York, USA (NMNH); Museo de Zoología de la Universidad de Concepción, Chile (MZUC = UCCC). Se utiliza la sigla MZUC para designar la Colección del Museo de Zoología de la Universidad de Concepción, la que es equivalente a la usada en otros trabajos por el segundo autor como UCCC.

Para obtener caracteres de morfología interna de la genitalia, se realizaron disecciones de ejemplares secos, previo aclarado en $\mathrm{NaOH}$, según la metodología usada por Artigas (1971) y Theodor
(1976). Las estructuras internas de la genitalia de los machos, fueron fotografiadas con un microscopio electrónico de barrido en el Laboratorio de Microscopía Electrónica de la Universidad de Concepción.

La distribución geográfica de las especies se estableció, en base a material recolectado, material perteneciente a colecciones nacionales y extranjeras y a los registros existentes en la literatura.

Se utiliza la nomenclatura alar y corporal propuesta por McAlpine (1981) y para la genitalia las abreviaturas utilizadas por Artigas (1995). Las especies $P h$. tibialis y $P h$. sagitata mencionadas en Hengst (2000), nunca fueron publicadas; éstas corresponden en este trabajo a Ph. amblayoensis y $P$ h. cienagaensis respectivamente, nombres que hacen alusión a las localidades de colecta de los tipos en cada caso.

\section{RESULTADOS}

Clave para las especies del género Philonerax Bromley

1. Machos 2

1'. Hembras .......................................................... 4

2. (1) Proyecciones terminales del esternito 8, en vista ventral, divergentes, forman un ángulo de $120^{\circ}$ y llevan abundantes cerdas largas, muy densas especialmente hacia el ápice (Fig. 2E) . mucidus Walker

2'. Tales proyecciones más o menos paralelas en toda su extensión (Fig. 4C y 6B) ...................... 3

3. (2') Fémures negros o con los extremos más claros; algunos como su Alotipo, con abundantes áreas claras amblayoensis sp. nov. 3'. Fémur longitudinalmente bicolor, negro, rojizo, al menos en los fémures anteriores y medianos cienagaensis sp. nov.

4. (1') Noveno tergito con una profunda hendidura en $\mathrm{V}$ en el centro del borde distal posterior (Fig. 6C y 6D) y dos a cuatro largos pelos cerdosos negros a cada costado, ligeramente abiertos hacia el costado, claramente diferenciados del resto de los pelos y cerdas del tergito

cienagaensis sp. nov.

4'. Noveno tergito con el borde posterior sólo con una débil invaginación o completo (Figs. 2D y $4 \mathrm{E})$; los pelos y cerdas son blancos y dirigidos hacia atrás 5

5. (4') Abdomen con una notable línea castaño oscura, desprovista de pelos, a lo largo de la línea medio dorsal mucidus Walker. 5'. Abdomen sin la línea mencionada arriba...... amblayoensis sp. nov. 
Philonerax mucidus (Walker, 1837)

Asilus mucidus Walker, 1837: 340.

Philonerax mucidus (Walker). Bromley, 1935: 270.

Lochmorhynchus mucidus (Walker). Artigas \& Papavero (1995): 134.

Lonchodogonus cribatus Hull, 1962: 498; Artigas \& Papavero, 1995: 134

Philonerax chilechicoensis Artigas, 1970: 344; Artigas \& Papavero, 1995: 133.

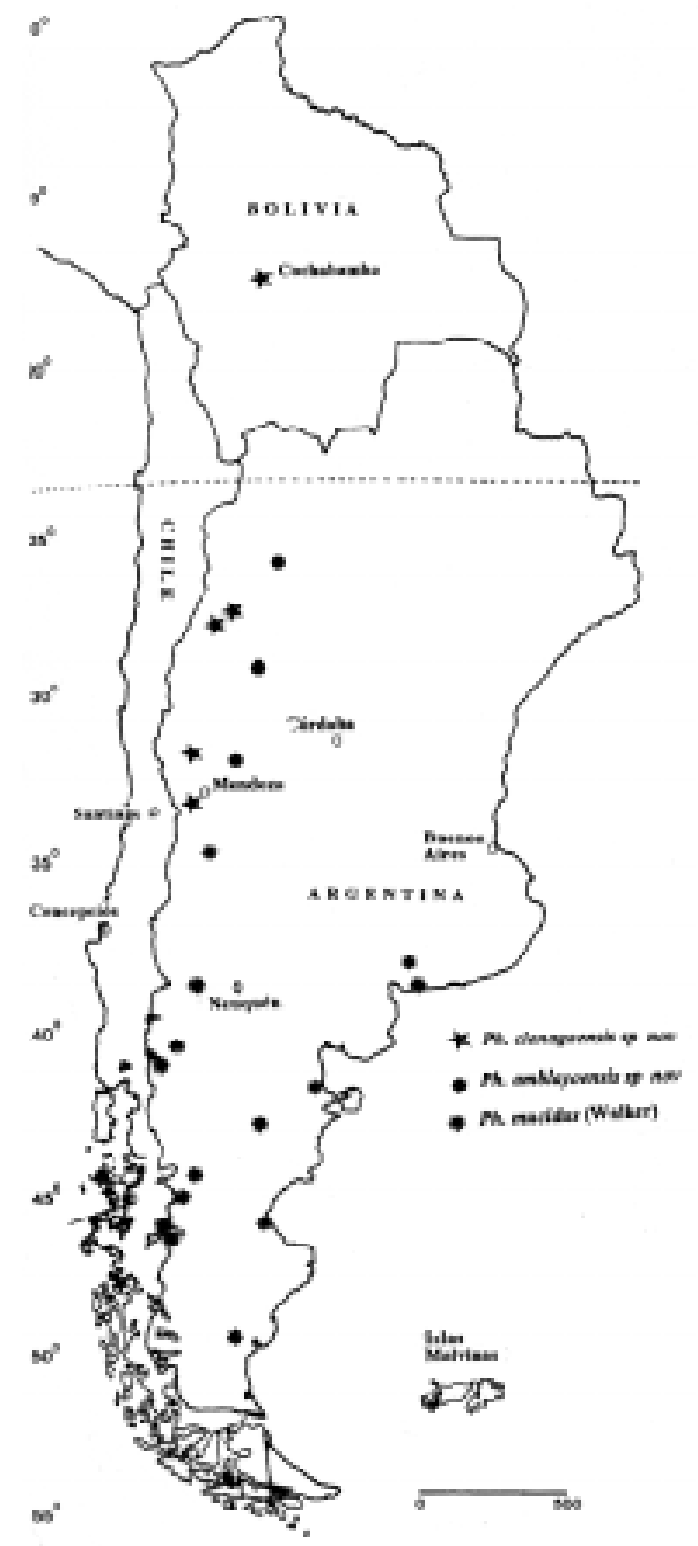

Fig. 1: Distribución de las especies del género Philonerax: Ph. amblayoensis sp. nov., Ph. cienagaensis sp. nov. y Ph. mucidus Walker.

Distribution of the species belonging to the genus Philonerax: Ph. amblayoensis sp. nov., Ph. cienagaensis sp. nov. and Ph. mucidus Walker.
Holotipo: $\sigma^{7}$, Port Sta Elena, Dec. Lieut. Graves. Depósito de este ejemplar, desconocido.

Longitud corporal: $11,0-18,1 \mathrm{~mm}$.

Longitud alar: 8,2-110,3 mm.

Diagnosis: cuerpo con tegumento castaño oscuro cubierto de polinosidad gris; pelos y cerdas blancas; pelos y cerdas de las antenas y mistax blancos; escutelo con cerdas blancas ordenadas en dos grupos que dejan la línea dorso central libre; fémur, al menos los anteriores y medianos, bicolor longitudinalmente negro y rojizo. Abdomen con una línea medio dorsal de color castaño, desprovista de la micropubescencia gris y pelos blancos que cubren el resto del abdomen. Machos con una expansión costa alar, las hembras carecen de ella. Genitalia negra, con pelos y cerdas blancas. Esternito octavo del macho grande (Fig. 2E), con dos extensiones aplanadas en su extremo posterior, las que se extienden hacia los costados divergentes en un ángulo de $120^{\circ}$, provistas en su extremo de largas cerdas blancas curvadas hacia el interior, cerrando posteriormente la cavidad genital. Las hembras son similares a los machos, tienden a tener más ancha la línea media dorsal del abdomen castaño oscura sin cerdas, el ovipositor es subesférico, precedido de una fuerte estrangulación formada por el ápice del $\operatorname{tg} 7$ y todo el octavo (Fig. 2D); el tergito noveno tiene el borde posterior recto; bordes del tg9 y cercos con abundantes cerdas blanco amarillentas, curvadas hacia abajo y al centro, las que cierran centralmente la abertura genital (Fig. 2B).

Material examinado: 28 ejemplares. En Argentina, Provincia de Chubut: Arroyo Pescado, 630 m, XI-19-1966, ME Irwin \& EI Schlinger cols, MZUC, $\left(\bigcirc^{7}\right.$ ). Chubut, Río Mayo, 20 noviembre 1966, MZUC (20); Arroyo Verde, 12 km S Río Lenguer, $710 \mathrm{~m}$, XI-20-66, EI Schlinger \& ME Irwin cols, MZUC $(1 \bigcirc)$. Provincia de Río Negro: Bariloche, 28-XI-I-XII-1926, F \& M Edwards BM cols, MZUC $(1 \odot, 1$ espermateca destruida). Territoro de Río Negro, F \& M Edwards BM cols, 1927-63, Lago Nahuel Huapi, Eastern End, 17XI-1926, MZUC $\left(1 \bigcirc^{\top}\right)$. Provincia de Mendoza: Rafael; LE Peña col, 23-X-87, MZUC $\left(10^{7}\right)$. Provincia de Neuquén: Mariano Moreno, XII-16-66, $750 \mathrm{~m}$ dunas, ME Irwin \& EI Schlinger cols, MZUC, $\left(1 \bigcirc^{7}\right)$. Provincia de Santa Cruz: $12 \mathrm{~km} \mathrm{~S}$ Bajo Caracoles, 640 m, XI-24 1966, EI Schlinger \& ME Irwin cols, MZUC (1@, 10 espermateca). Santa Cruz, 2 km S Caleta Olivia, 10 m, XII-121967, EI Schlinger \& ME\# Irwin cols, "coastal dune", MZUC, (4ᄋ, 1 ᄋ espermateca); 5,6 km E Los Antiguos, $360 \mathrm{~m}$, XI-23-66, EI Schlinger \& ME Irwin cols, MZUC, $(1 \&) ; 5 \mathrm{~km} \mathrm{NW}$ Piedrabuena, XI-25-66, $130 \mathrm{~m}$, EI Schlinger \& ME Irwin cols, MZUC $(1 \bigcirc) ; 2,8$ km E Los Anti- 
guos, $280 \mathrm{~m}$, XI-21-1967, EI Schliunger \& ME Irwin cols, MZUC (1@, $1 \bigcirc^{7)}$.

En Chile, Región de Aysén, 250 m, Chile Chico, XI-21-1966, EI Schlinger \& ME irwin cols,

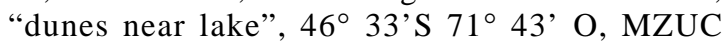
(1@). Aysén, Puerto Ibáñez, Laguna Buenos Aires, 12-15-I-61, LE Peña col, MZUC (1 $\left.\bigcirc^{7}\right)$, holotipo de Ph. chilechicoensis Artigas); Laguna Buenos Aires, 24-31-XII-60, LE Peña col, MZUC, (1), Alotipo Ph. chilechicoensis Artigas, 1 , espermateca); $250 \mathrm{~m}$, Chile Chico, XI-21-1966, EI Schlinger \& ME Irwin cols, "dunes near lake", $46^{\circ} 35^{\prime} \mathrm{S} 71^{\circ} 43^{\prime} \mathrm{O}, \mathrm{MZUC}\left(1 \bigcirc, 1 \bigcirc^{\prime}\right)$.

\section{Philonerax amblayoensis sp. nov.}

Diagnosis: tegumento del cuerpo pruinoso, castaño grisáceo, con predominio de pilosidad amarillenta o blanco amarillenta brillante. Algunos ejemplares pueden ser predominantemente ne- gros, aunque domina la tendencia de fémures negros o bicolor y tibias amarillas. Se distingue de Ph. mucidus (Walker), su especie mas próxima, por tener las proyecciones posteriores del esternito 8 dirigidos hacia atrás en forma paralela y por los pliegues membranosos de la base interna de los gonopodos de los machos.

Holotipo: Argentina, Salta, Alturas de Amblayo, $3.500 \mathrm{~m}$ de altitud, 5 abril 1985, Col. Fritz, MZUC.

Longitud corporal: $16,0 \mathrm{~mm}$.

Longitud alar: $10,5 \mathrm{~mm}$.

Cabeza: en vista frontal es circular, 1/5 más ancha que alta. Ocelos amarillos. Cerdas ocelares negras; frontales blancas. Frente angosta, cubierta de micropubescencia plateada; antenas muy juntas, casi sin espacio entre los soquetes de inserción. Espacio entre las antenas y el tubérculo ocelar glabro y brillante. En vista lateral: Cerdas postoculares amarillas, con pelos blancos largos. Cerdas y pelos occipitales blanco amarillen-

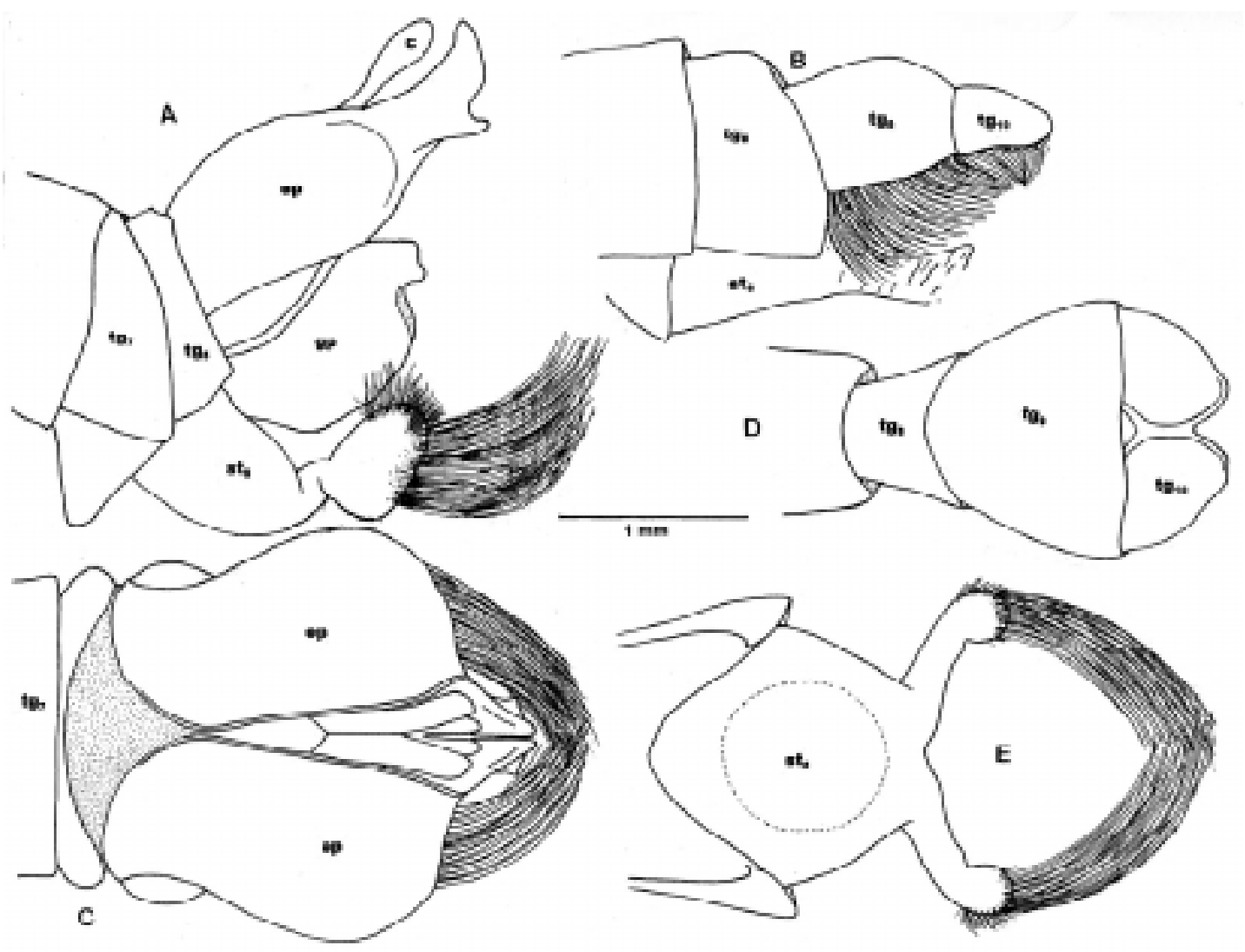

Fig. 2: Philonerax mucidus (Walker): (A) macho, vista lateral; (B) hembra, vista lateral; (C) macho, vista dorsal; (D) hembra, vista dorsal; (E) macho, vista ventral. Clave de abreviaciones: (ep) epandrio, $(\mathrm{gp})$ gonopodo, $\left(\mathrm{st}_{8}\right)$ octavo esternito, $\left(\mathrm{tg}_{7}\right)$ séptimo tergito, $\left(\operatorname{tg}_{8}\right)$ octavo tergito, $\left(\operatorname{tg}_{9}\right)$ noveno tergito, $\left(\operatorname{tg}_{10}\right)$ décimo tergito.

Philonerax mucidus (Walker): (A) male, lateral view; (B) female, lateral view; (C) male, dorsal view; (D) female, dorsal view; (E) male, ventral view. Key to abbreviations: (ep) epandria, (gp) gonopod, $\left(\mathrm{st}_{8}\right)$ eight sternite, $\left(\operatorname{tg}_{7}\right)$ seventh tergite, $(\operatorname{tg} 8)$ eight tergite, $\left(\operatorname{tg}_{9}\right)$ ninth tergite, $\left(\operatorname{tg}_{10}\right)$ tenth tergite. 
tas. Antenas negras, escapo y pedicelo con cerdas negras en el dorso y costados, el escapo con pelos cerdosos ventrales blancos más largos; escapo largo, igual a dos veces la longitud del pedicelo y de igual longitud que el primer flagelómero; primer flagelómero antenal largo, cubierto de micropubescencia gris amarillenta; en el dorso hay algunas microcerdas; flagelo antenal negro, ligeramente ensanchado antes del ápice, terminando en una punta aguda. Mystax blanco amarillento uniforme, las cerdas del borde oral son blancas. Cerdas de los palpos negras;.proboscis negra brillante, con pelos blanco amarillentos en el borde ventral.

Tórax: vestidura del tórax con áreas de pelos castaño grisáceos y gris blanquecino. Cerdas del pronoto amarillas, entremezcladas con pilosidad rala de igual longitud y más clara. Mesonoto con pelos negros, que aumentan en longitud por detrás de la sutura transversa y alcanzan la misma longitud que las cerdas dorsocentrales que también son negras; líneas medio-dorsales, más anchas en la parte anterior; tórax cubierto con micropubescencia dorada, excepto en las dos líneas medio-dorsales y el escutelo, en que ésta está ausente. Tres cerdas notopleurales amarillas y una cerda intraalar y dos postalares negras, acompañadas de otras más finas, también negras (en algunos paratipos castaño rojizas). Callos humerales con largos pelos cerdosos blancos y negros. Escutelo con cerdas blanco amarillentas predominantes hacia los bordes y otras más oscuras en el disco; además hay algunas cerdas negras, más gruesas que se disponen en el borde central posterior.

Alas: alas hialinas, con una leve expansión costal (Fig. 3E). Venas castaño oscuras.

Patas: coxas y fémures negro brillantes, tibias anteriores castaño rojizas. Fémur anterior con pilosidad corta en el dorso y más larga hacia los

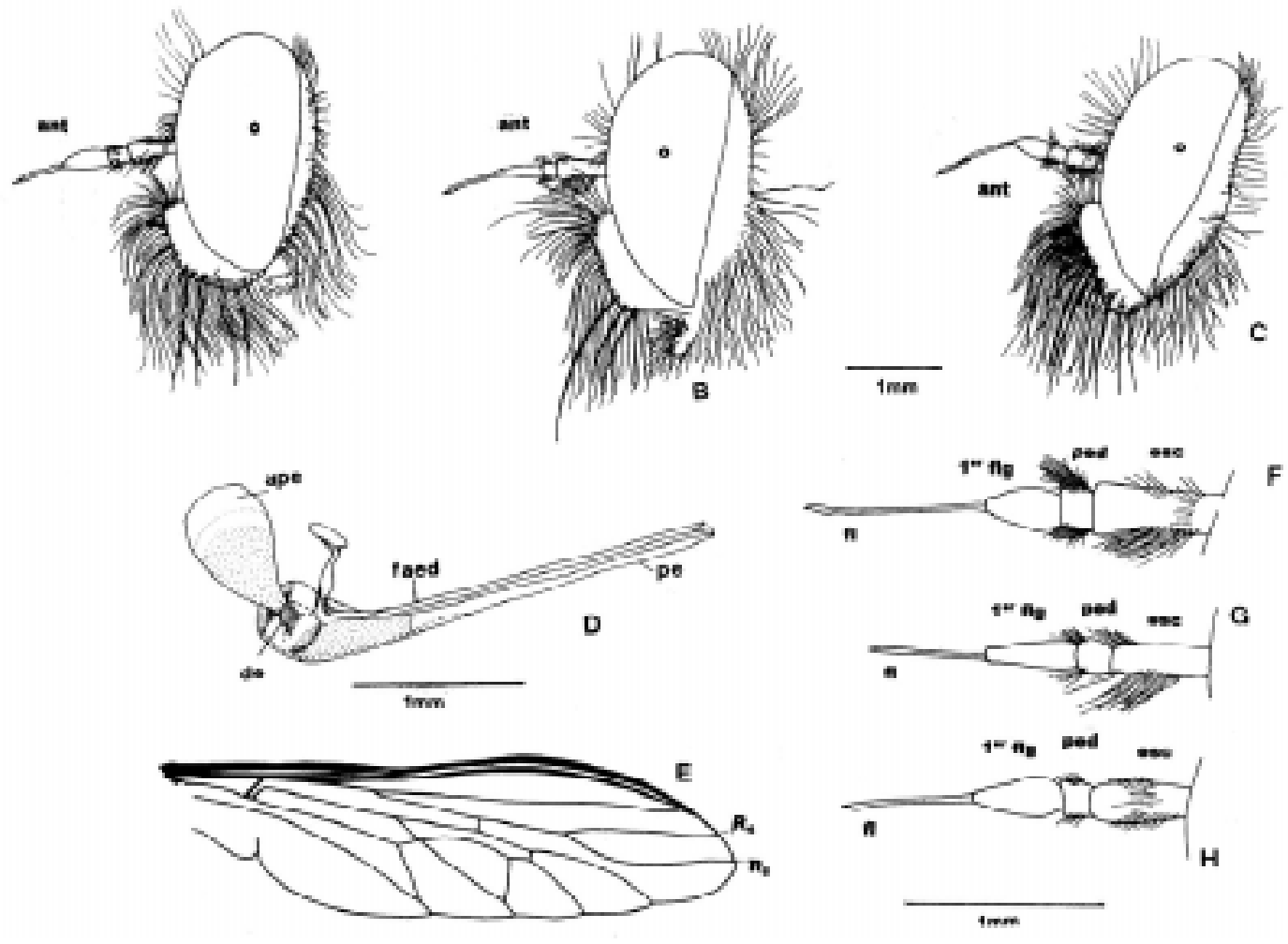

Fig. 3: Philonerax cienagaensis sp. nov: (A) cabeza, vista lateral; (B) Ph. amblayoensis sp. nov. Cabeza vista lateral; (C) Ph. mucidus, cabeza, vista lateral; (D) Ph. ablayoensis, Aedeagus; (E) Ph. amblayoensis, ala; (F) Ph. mucidus, antena; (G) Ph. ablayoensis, antena; (H) Ph. cienagaensis, antena. Clave de abreviaciones: (ant) antena, (o) ojo, (ape) apodema eyaculador, (de) ducto eyaculador, (caed) aedeagus, (pe) procesos expulsores, (ped) pedicelo, (esc) escapo, ( $\left.1^{\mathrm{er}} \mathrm{flg}\right)$ primer flagerómero antenal, $\left(\mathrm{R}_{4}\right)$ cuarta vena radial, $\left(\mathrm{r}_{5}\right.$ ) quinta vena radial.

Philonerax cienagaensis sp. nov; (A) head, lateral view; (B) Ph. amblayoensis sp. nov. head, lateral view; (C) Ph. mucidus head, lateral view; (D) Ph. amblayoensis, aedeagus; (E) Ph. amblayoensis, Wing; (F) Ph. mucidus, antenna; (G) Ph. cienagaensis, antenna. Key to abbreviations: (ant) antenna, (o) eye, (ape) ejaculator apodem, (de) ejector duct, (faed) aedeagus, (pe) ejector process, (ped) pedicelo, (esc) escapo, $\left(1^{\text {er }}\right.$ flg) first flagelomere, $\left(R_{4}\right)$ forth radial vein, $\left(R_{5}\right)$ fifth radial vein. 
lados y ventralmente, sin cerdas gruesas; fémur mediano, con pilosidad similar al fémur anterior, con cerdas amarillas cortas, fémur posterior, con pilosidad blanca, corta en los lados y en el dorso; ventralmente lleva pelos más largos, principalmente de color castaño oscuros o negros (en paratipos, los fémures medianos y posteriores pueden presentar cerdas espinosas negras entre las amarillas). Tibias con largos pelos blancos y algunos negros entremezclados; cerdas amarillas, largas, además de algunas cerdas negras. La región ventral de las tibias con largo pelo cerdoso, blanco amarillento. Tarsos con pilosidad similar a la de las tibias; con dos largas cerdas blanco amarillentas a cada lado, en los tarsómeros; uñas curvadas y agudas.

Abdomen: abdomen cilíndrico, de aspecto castaño pruinoso, más notable en los tergitos 5 al 7.
Pilosidad del abdomen corta y dura, blanca a blanca amarillenta; con pelos largos en todos los tergitos, más largos en los tergitos 1 al 3. Tergito 1 con cerdas amarillas fuertes a cada lado; tergitos 2 al 4 en la región dorsal, grisáceos, sin pruina castaño amarillenta. Esternitos cubiertos de pruina castaño amarillenta.

Genitalia: genitalia pequeña, castaño rojiza, con pilosidad del mismo color que el resto del cuerpo. En vista lateral los epandrios (Figs. 4H) son angostos antes del ápice y espatulados en el extremo. (en paratipos aclarados en $\mathrm{KOH}$, se observa por transparencia, una hilera de cortas espinas negras en el borde interno, (Fig. 4H). Gonopodo como en la Fig. 4G; (en paratipos se observa por microscopía de barrido, pliegues en la base interna de la mitad posterior). Hypandrio corto, oculto debajo del octavo esternito, escasa-
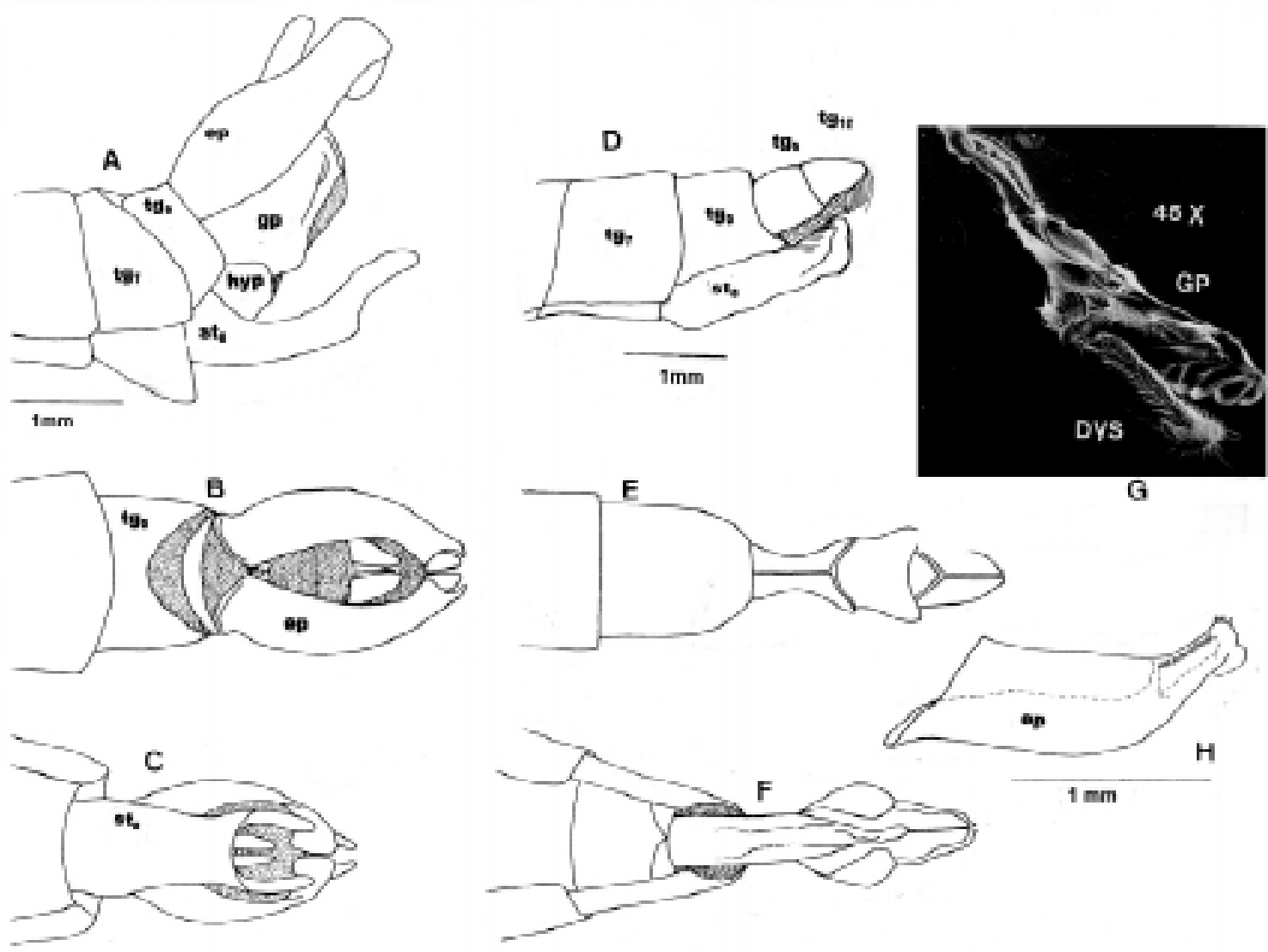

Fig. 4: Philonerax amblayoensis sp. nov.: (A) macho, vista lateral; (B) macho, vista dorsal; (C) macho, vista ventral; (D) hembra, vista lateral; (E) hembra, vista dorsal; (F) hembra, vista ventral; (G) macho, gonopodo; (H) macho, epandrio. Clave de abreviaciones: (dys) dystistili, (ep) epandrio, (gp) gonopodo, $\left(\mathrm{st}_{8}\right)$ octavo esternito, $\left(\operatorname{tg}_{7}\right)$ séptimo tergito, $\left(\operatorname{tg}_{8}\right)$ octavo tergito, $\left(\operatorname{tg}_{9}\right)$ noveno tergito, $\left(\operatorname{tg}_{10}\right)$ décimo tergito.

Philonerax amblayoensis sp. nov.: (A) male, lateral view; (B) male, dorsal view; (C) male, ventral view; (D) female, lateral view; (E) female, dorsal view; (F) female, ventral view; (G) male, gonopod; (H) male, epandria. Key to abbreviations: (dys) dystistili, (ep) epandria, (gp) gonopod, $\left(\mathrm{st}_{8}\right)$ eight sternite, $\left(\operatorname{tg}_{7}\right)$ seventh tergite, $\left(\operatorname{tg}_{8}\right)$ eight tergite, $\left(\operatorname{tg}_{9}\right)$ ninth tergite, $\left(\operatorname{tg}_{10}\right)$ tenth tergite. 
mente visible lateralmente. Esternito 8 en vista ventral alargado, igual a 1,5 veces la longitud de las largas proyecciones apicales paralelas (Fig. 4C) que llevan largos pelos blanco amarillentos que no alcanzan a cerrar por abajo la cavidad genital como en Ph. mucidus. Aedeagus como en la Fig. 3D.

Alotipo: $\bigcirc$, Salta, Alturas de Amblayo, $3.500 \mathrm{~m}$ de altuitud, abril, Fritz col. MZUC. Similar al holotipo en tamaño, color y cubierta pilosa. Las alas no tienen expansión costal. El fémur anterior de la hembra, presenta fuertes cerdas de color blanco amarillentas, ausentes en los machos; el fémur posterior de las hembras no posee pelos negros.

Genitalia de la hembra: octavo segmento abdominal fuertemente comprimido (Fig. 4E). Luego se ensanchan los tergitos 9 y 10 , formando una

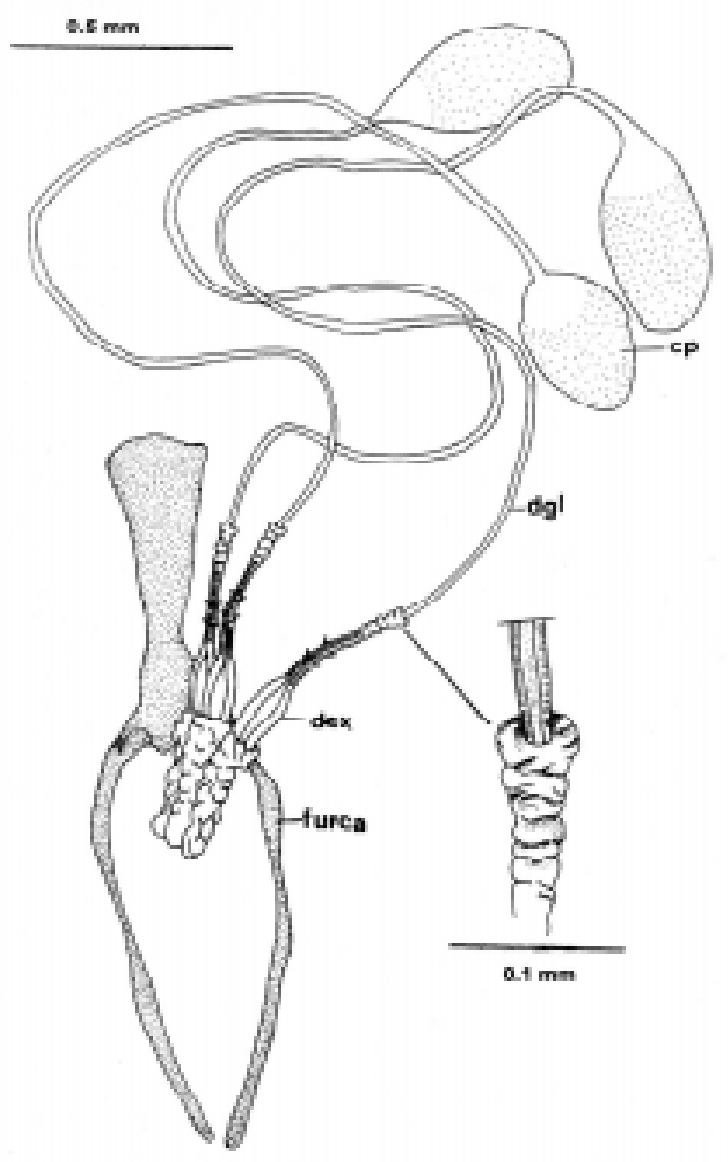

Fig. 5: Philonerax amblayoensis sp. nov., espermateca. Clave de abreviaciones: (cp) cápsula, (dgl) ducto glandular, (dex) ducto expulsor.

Philonerax amblayoensis sp. nov., spermatheca. Key to abbreviations: (cp) capsule, (dgl) glandular duct, (dex) expeller duct. estructura en forma de cuenta, de los cuales penden pelos largos, dorados que cierran por debajo la genitalia a manera de un canastillo. Esternito 8 en vista lateral con forma de quilla (Fig. 4D) que alcanza hasta el décimo tergito, sin pasar más allá del ápice del abdomen, con la misma vestidura del resto del abdomen. Espermateca como en la Fig. 5.

Distribución geográfica: Argentina, Provincia de La Rioja, Salta y San Juan (Fig. 1).

Origen del nombre: el nombre específico hace alusión a la localidad del tipo, Argentina, Provincia de Salta, Alturas de Amblayo.

Material examinado: 10 ejemplares. En Argentina, Provincia de La Rioja: La Rioja, $40 \mathrm{~km} \mathrm{E}$ Chepes, abril (2○ y $\left.1 \bigcirc^{7}\right)$ Peña y Barría col. MZUC. Provincia de Salta: Salta, Alturas de Amblayo, $3.500 \mathrm{~m}$, abril (1 ej. holotipo, 2 e) Fritz col. MZUC. Provincia de San Juan: 40 km E San Juan, abril (1 ej. Alotipo, 1 e) Peña y Barría col. MZUC; San Juan, 82 km E San Juan, abril (1 ej.) Peña y Barría col. MZUC; San Juan, 100 km E San Juan, abril (1 ej. ) Peña y Barría col. MZUC.

Philonerax cienagaensis sp. nov.

Diagnosis: tegumento castaño rojizo, con pruina cenicienta, irregularmente distribuida, vestidura de finos pelos cortos y ralos; cerdas del cuerpo en su mayoría negras. Alas hialinas; con expansión costal alar en el macho, algunos con manchas café cerca de la expansión costal. Se distingue de $P h$. mucidus (Walker) y $P h$. amblayoensis sp. nov. por presentar los fémures longitudinalmente bicolor, negro y rojizo. En las hembras hay 3-5 gruesas cerdas largas y negras en los bordes internos del noveno tergito, el que está fuertemente sagitado (Fig. 6D).

Holotipo: ${ }^{7}$, San Juan, La Ciénaga, Argentina, March 6. 1992. L.E.Parra. Leg. MZUC.

Longitud corporal: $17,8 \mathrm{~mm}$

Longitud alar: $11,5 \mathrm{~mm}$

Cabeza: en vista frontal es sub-cuadrandrangular (levemente más ancha que alta). Cerdas ocelares negras y largas, las frontales negras, (o blancas y negras entremezcladas en algunos paratipos). En vista lateral: cerdas postoculares blancas y negras. Antenas con escapo y pedicelo castaños, (en algunos paratipos son oscuros), con cerdas blancas en el dorso, y blancas y castañas entremezcladas, más largas en la zona ventral; primer flagelómero largo, atenuado hacia el ápice; flagelo 1,5 veces el primer flagelómero; hacia el ápice presenta una hendidura que lo hace más angosto para luego terminar en punta. Giba facial negra, cubierta de micropubescencia plateada igual que 
la frente y detrás de los ojos. Barba blanca. Mystax con cerdas blancas y negras entremezcladas; cerdas del borde oral y de los palpos maxilares blancas. Barba blanca.

Tórax: la cobertura es rala, deja ver el tegumento castaño con tintes rojizos, con una línea mediodorsal negra, gruesa, en el dorso con dos manchas laterales del mismo color. Cerdas muy cortas, negras en el dorso y blancas y negras en los costados; dos cerdas notopleurales, una intraalar y dos postalares. Callos humerales con finas cerdas negras cortas y, pelos blancos largos. Región posterodorsal del tórax desnuda, castaño rojiza, con escasa micropubescencia plateada. Escutelo con pelos cerdosos blancos y negros en el disco y, cerdas fuertes hacia el borde.

Alas: las alas (Fig. 4E) hialinas, pero con ligero tinte castaño, a la altura de la expansión costal; venas castaño oscuras.

Patas: coxas longitudinalmente bicolor, con la mitad anterior negra y la posterior rojiza, con escasa micropubescencia gris y finos pelos largos y cerdas más gruesas, todos blancos. Fémures y tibias longitudinalmente bicolor, negro rojizo, cubiertos de pelos blancos, más largos en el fémur anterior. Cerdas blancas y negras en los fémures, negras en las tibias; fémur anterior con pilosidad predominantemente blanca, larga y fina; fémur posterior, con pelos largos negros y castaños, finos, en la parte ventral. Cerdas de los fémures blancas y negras, en las tibias principalmente negras. Tarsómeros castaños, con pelos blancos, similares al resto de la pata; las cerdas son negras fuertes, dos dorsales y dos a cada lado más largas negras, las ventrales más cortas. Uñas agudas y largas, negras, de base rojiza.

Abdomen: abdomen cubierto de pruina cenicienta, que predomina en los bordes, anterior, posterior y laterales. Tergito 1 amarillo pálido, con pelos blancos y negros dispersos; tergitos 6 y 7 casi totalmente cubiertos de pruina cenicienta; tergito 6 más ancho hacia el borde distal; tergito 7 muy corto. Cobertura pilosa con cerdas negras, cortas y ralas dorsalmente, y blancas y más lar-

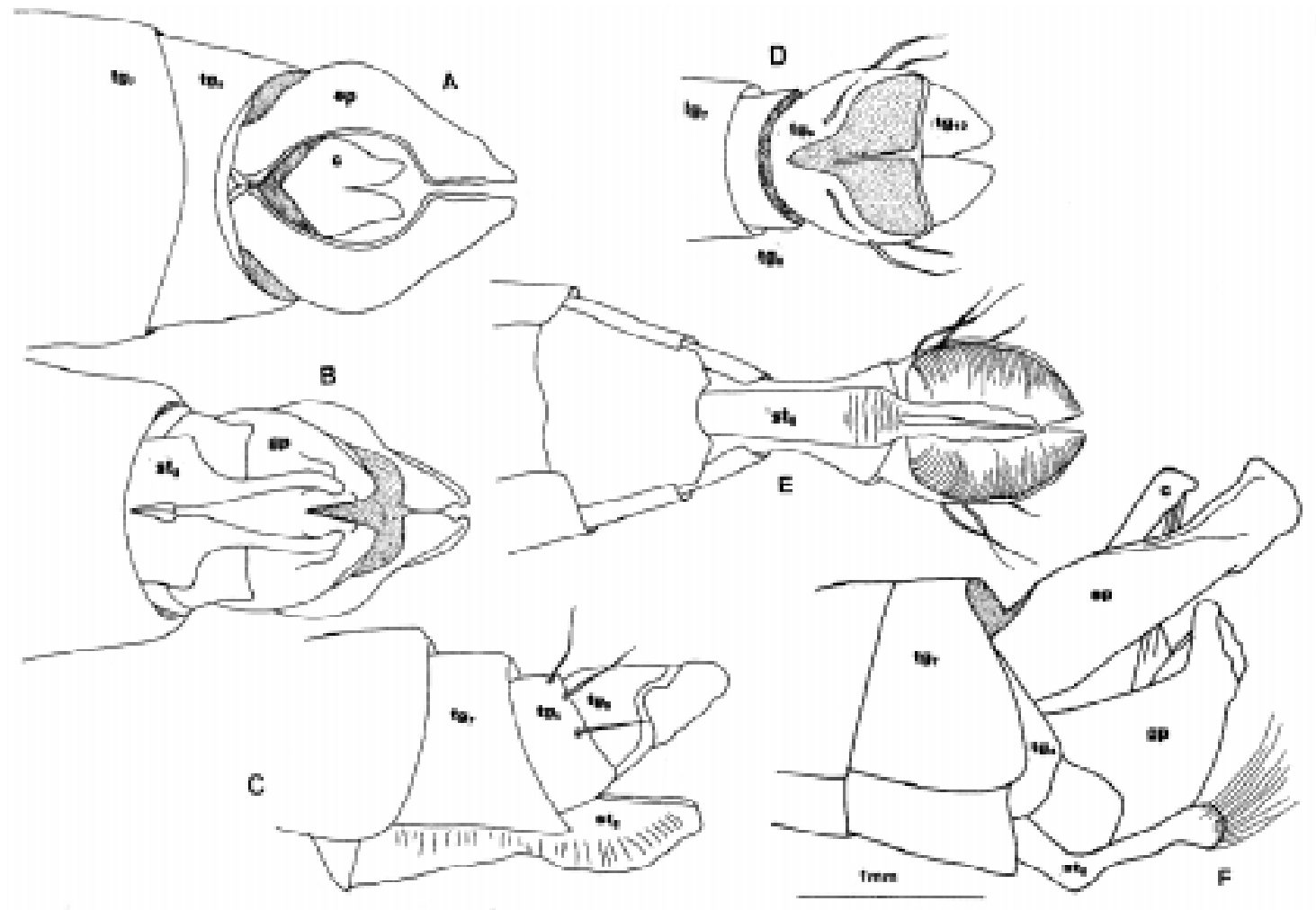

Fig. 6: Philonerax cienagaensis sp. nov.: (A) macho, vista dorsal; (B) macho, vista ventral; (C) hembra, vista lateral; (D) hembra, vista dorsal; (E) hembra, vista ventral; (F) macho, vista lateral. Clave de abreviaciones: (c) cercos, (ep) epandrio, (gp) gonopodo, $\left(\mathrm{st}_{8}\right)$ octavo esternito, $\left(\operatorname{tg}_{7}\right)$ séptimo tergito, $\left(\operatorname{tg}_{8}\right)$ octavo tergito, $\left(\operatorname{tg}_{9}\right)$ noveno tergito, $\left(\operatorname{tg}_{10}\right)$ décimo tergito.

Philonerax cienagaensis sp. nov.: (A) male, dorsal view; (B) male, ventral view; (C) female, lateral view; (D) female, dorsal view; (E) female, ventral view; (F) male, lateral view. Key to abbreviations: (c) cerci, (ep) epandria, (gp) gonopod, $\left(\mathrm{st}_{8}\right)$ eight sternite, $\left(\operatorname{tg}_{7}\right)$ seventh tergite, $\left(\operatorname{tg}_{8}\right)$ eight tergite, $\left(\operatorname{tg}_{9}\right)$ ninth tergite, $\left(\operatorname{tg}_{10}\right)$ tenth tergite. 


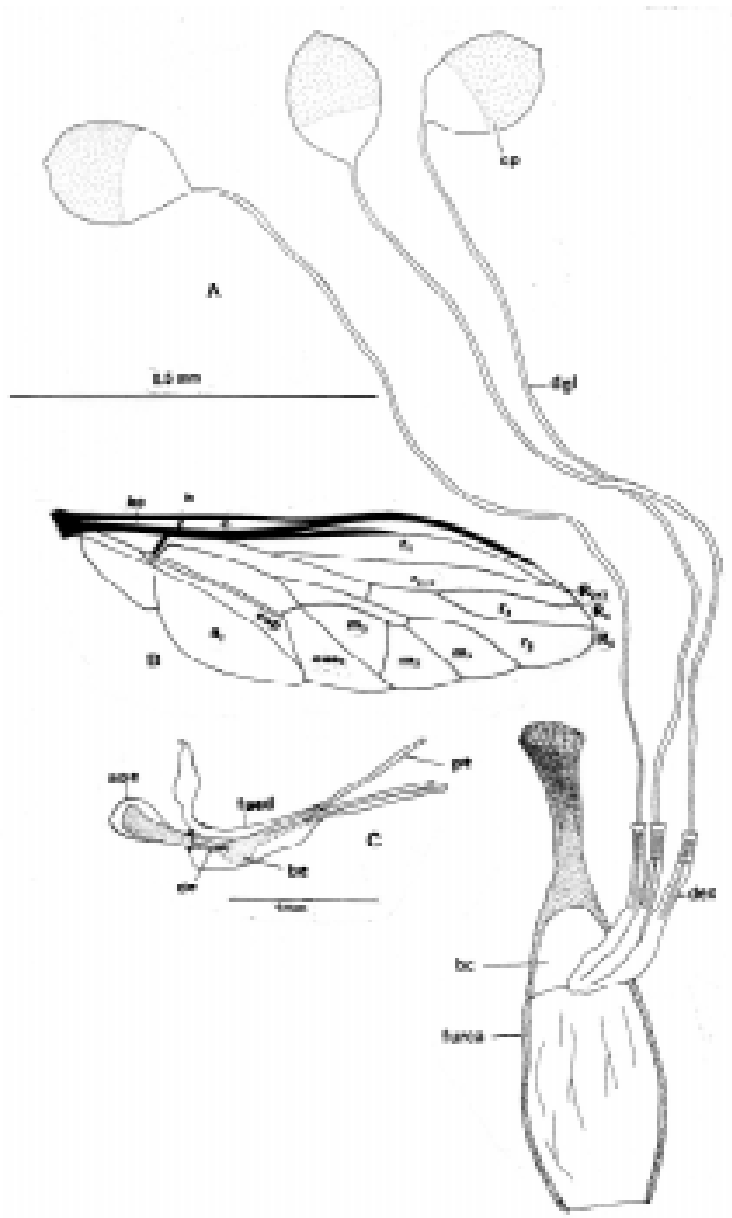

Fig. 7: Philonerax cienagaensis; (A) hembra, espermateca; (B) macho, ala; (C) macho (aedeagus). Clave de abreviaciones: (ape) apodema eyaculador, (bc) bursa copulatrix, (be) base del aedeagus, (cp) cápsula, (dgl) ducto glandular, (dex) ducto expulsor, (faed) aedeagus, (pe) procesos expulsores, $\left(a_{1}\right)$ primera celda anal, (bc) celda basal costal, (h) vena transversa humeral, (c) celda costal, $\left(r_{1}\right)$ primera celda radial, $\left(\mathrm{r}_{2+3}, \mathrm{r}_{4}\right)$ cuarta celda radial, $\left(\mathrm{r}_{5}\right)$ quinta celda radial, $\left(\mathrm{m}_{1}\right)$ primera celda radial, $\left(\mathrm{m}_{2}\right)$ segunda celda radial, $\left(\mathrm{m}_{3}\right)$ tercera celda medial, (cup) celda posterio cubital, $\left(\mathrm{R}_{4}\right)$ cuarta vena radial, $\left(\mathrm{R}_{5}\right)$ quinta vena radial, $\left(R_{2+3}\right)$ primera y segunda vena radial fusionadas.

Philonerax cienagaensis; (A) female, spermatheca; (B) male, wing; (C) male, aedeagus. Key to abbreviations: (ape) ejaculator apodem, (bc) bursa copulatrix, (be) base of aedeagus, (cp) capsule, (dgl) glandular duct, (dex) expeller duct, (faed) aedeagus, (pe) ejector process, $\left(a_{1}\right)$ first anal cell, (bc) costal basal cell, (h) transversal humeral vein, (c) costal cell, $\left(r_{1}\right)$ first radial cell, $\left(r_{4}\right)$ forth radial cell, $\left(r_{5}\right)$ fifth radial cell, $\left(\mathrm{m}_{1}\right)$ first medial cell, $\left(\mathrm{m}_{2}\right)$ second medial cell, $\left(\mathrm{m}_{3}\right)$ third medial cell, (cup) posterior cubital cell, $\left(\mathrm{R}_{4}\right)$ forth radial vein, $\left(\mathrm{R}_{5}\right)$ fifth radial vein, $\left(\mathrm{R}_{2+3}\right)$ first and second radial veins united. gas, uniformemente distribuidas en la parte ventral. Borde distal de los tergitos 2 al 5 de coloración anaranjada sin pelos.

Genitalia: bicolor negro y rojizo, subglobosa en vista dorsal (Fig. 4G y 6A). Los epandrios son aplanados en el ápice y con sus bordes posteriores en contacto, en el área mediana llevan algunos pelos cerdosos blancos. Octavo esternito (Fig. 6B) con dos procesos largos, casi paralelos, separados en forma de $\mathrm{V}$ (ángulo agudo), con cerdas blancas en el ápice. Hypandrio visible en vista lateral. Aedeago como en la Fig. 7C.

Alotipo: O, Argentina, La Ciénaga, San Juan, marzo 6, 1992. L.E. Parra. Leg. MZUC. Igual al macho, las alas sin expansión costal.

Genitalia de la hembra: abdomen cónico. Tergito 8 fuertemente comprimido; noveno con una profunda escotadura, desde donde nacen, en el borde distal, 4 a 5 cerdas largas, gruesas y negras (Fig. 5). En los bordes de los tergitos 9 y 10, hay pelos amarillo dorados, muy juntos, dirigidos hacia abajo, que tienden a cerrar centralmente la cavidad genital. Esternito 8 en forma de quilla en la mitad apical, la mitad basal es centralmente aplanada. Espermateca como en la Fig. 7A.

Distribución geográfica: Argentina (Provincia de Catamarca, Provincia de Mendoza, Provincia de San Juan y Provincia de Tucumán) y Bolivia (Departamento de Cochabamba) (Fig. 1).

Origen del nombre: el nombre específico hace alusión a la localidad tipo, Argentina, La Ciénaga, Provincia de San Juan.

Material examinado: 8 ejemplares. En Argentina, Provincia de Catamarca: Punta Balasto, Catamarca, febrero (2 ej. paratipos) Peña col. MZUC. Provincia de Mendoza: $17 \mathrm{~km}$ SO Mendoza, noviembre (1 ej. paratipo) A. Ugarte col. MZUC. Provincia de San Juan: San Juan, La Ciénaga, marzo (3 ej. paratipos) Peña col. MZUC. Provincia de Tucumán: Tucumán, La Mesada, Departamento de Buruyac, octubre (1 ej. paratipo) Ares col. MZUC.

En Bolivia, Departamento de Cochabamba: Capinota, enero (2 ej.) H. Merable col. Smithsonian Instution, NMNH.

\section{DISCUSIÓN}

Las especies del género Philonerax son muy similares entre sí. Los machos son más difíciles de separar, ya que la mayoría de las estructuras de valor taxonómico de su genitalia son visibles sólo previo aclarado en $\mathrm{NaOH}$. El octavo esternito del macho si bien constituye un carácter variable para separar especies dentro de Asilinae (Theodor, 1980), en Philonerax presenta un patrón genérico notable debido a la longitud de sus proyecciones 
distales, longitud de la base y la pilosidad, lo que permite separar con facilidad las especies del género hasta ahora conocidas. Las hembras de las especies $P h$. amblayoensis sp. nov. y $P h$. cienagaensis sp. nov, son separadas principalmente a partir de caracteres de la genitalia, tales como la escotadura del noveno tergito y la presencia de cerdas gruesas en el borde interno del noveno tergito presente solo en Ph. cienagaensis sp. nov.

\section{AGRADECIMIENTOS}

Este trabajo fue financiado parcialmente por el proyecto DIUC (Dirección de Investigación de la Universidad de Concepción). Apoyo Gestión FONDECYT No. 97.113.043-1.2. Además se agradece al señor Alfredo Ugarte por su aporte en ejemplares de asílidos, donados a nombre de Luis Peña a la colección del Museo de Zoología de la Universidad de Concepción.

\section{LITERATURA CITADA}

ARTIGAS JN (1970a) Los asílidos de Chile (DipteraAsilidae). Gayana Zoología (Chile) 17: 19-56 y 281 295.

ARTIGAS JN \& N PAPAVERO (1988) The American genera of Asilidae (Diptera): keys for identification with an atlas of female spermathecae and other morphological details. I. Key to subfamilies and subfamily Leptogastrinae Schiner. Gayana Zoología (Chile) 52: 95-114.

ARTIGAS JN \& N PAPAVERO (1995) The American genera of Asilidae (Diptera): keys for identification with an atlas of female spermathecae and other morphological details. IX. 5. subfamily Asilinae Leach, LOCHMORHYNCHUS-Group, with a catalogue of the Neotropical species. Gayana Zoología (Chile) 59: 131-144.
ARTIGAS JN \& MB HENGST (1998) Asilidae. En: Morrone JJ \& S Coscarón (eds) Biodiversidad de Artrópodos Argentinos: 353-364. Ediciones Sur, La Plata, Argentina.

ARTIGAS JN \& MB HENGST (1999) Clave ilustrada para los géneros de asílidos argentinos. Revista Chilena de Historia Natural 72: 107-150.

BROMLEY S (1932) Diptera of Patagonia and south Chile. British Museum of Natural History, London 5: 269-272.

HENGST MB (2000) Lochmorhynchus Engel, 1930 sensu lato (Diptera: Asilinae): taxonomía e inferencias filogenéticas. Tesis de Grado, Facultad de Ciencias Naturales y Oceanografícas, Universidad de Concepción, Concepción, Chile. ix +227 pp.

HULL F (1962) Robber flies of the world. The genera of the family Asilidae. Part 2. United States National Museum Bulletin 224: 433-907.

MCALPINE J (1981) Manual of Nearctic Diptera. Department of Agriculture Research Branch, Ottawa, Ontario, Canada. Volume 1.674 pp.

MACQUART MJ (1850) Histoire Naturelle: Diptères Exotiques nouveaux ou peu connus. Bulletin Museum Historie Naturelle de Paris (Supplément 4): 82-85.

PHILLIPPI RA (1865) Aufzählung der chilenischen Dipteren. Wien Zoologishe Botanische Gesellschaften 15: 595-782.

WALKER F (1837) Description of Diptera collected by Capt. King in the Survey of Straits of Magellan. Transactions of the Linnean Society of London 17: 340-341.

WOOD GC (1981) Asilidae. In: McAlpine JF et al. (eds) Manual of Nearctic Diptera 1: 549-573. Department of Agriculture Research Branch, Ottawa, Ontario, Canada. 